\title{
Modeling and Simulation of Electrical Impedance Tomography (EIT) on Ventilated Patients with ARDS Lungs
}

\author{
Mouloud Denaï, Mahdi Mahfouf, and Gary H. Mills
}

\begin{abstract}
Bedside monitoring of lung recruitment is a key feature in the ventilatory management of patients with acute respiratory distress syndrome. Electrical Impedance Tomography is a relatively new imaging technique which reconstructs the electrical conductivity distribution in a lung cross section from electrical measurements made on electrodes placed on the surface of the thorax. The induced conductivity changes can be continuously monitored to assess the topographical distribution of air in the lung and optimise the ventilation strategy. The purpose of this paper is to illustrate these principles on a physiological model of the lung mechanics simulating the concept of recruitment in ventilated patients with acute respiratory distress syndrome.
\end{abstract}

\section{INTRODUCTION}

A CUTE Respiratory Distress Syndrome (ARDS) is a sudden, life-threatening lung dysfunction associated with severe and diffuse alveolar damage and increased microvascular permeability leading to pulmonary edema and heterogeneous lung density redistribution [1], [2]. Compliance of either lung is decreased causing increased work of breathing (WOB) and subsequent respiratory The fundamentals of therapy are the provision of lung rest and supportive breathing in the Intensive Care Unit (ICU) using mechanical ventilation with a careful attention to fluid balance. Mechanical ventilation may, however, result in secondary lung damage referred to as Ventilator-Induced Lung Injury (VILI) caused by barotraumas (repetitive and reopening of injured alveoli) and/or pulmonary over distension when the lung units are physically stretched beyond their normal maximum inflation point.

Positive End Expiratory Pressure (PEEP) is the most effective way to achieve adequate oxygenation in ARDS patients. It improves the Functional Residual Capacity and prevents recruited alveoli to collapse. However very PEEP may decrease venous return and cardiac output and increase the risk of the development of extra pulmonary air (eg. Pneumothorax) [3]. Low Tidal Volumes $\left(V_{t}\right)$ is another effective standard of ventilator therapy in patients with ARDS [4]. Ideally, mechanical ventilation should be conducted with sufficient PEEP to prevent end- expiratory

M. Denaï is with the Department of Automatic Control and Systems Engineering, The University of Sheffield, Mappin Street, Sheffield S1 3JD, UK (e-mail: m.denai@ shef.ac.uk).

M. Mahfouf, is with the Department of Automatic Control and Systems Engineering, The University of Sheffield, Mappin Street, Sheffield S1 3JD, UK (e-mail: m.mahfouf@ shef.ac.uk).

G. H. Mills is with Intensive Care Unit, Royal Hallamshire Hospital, Glossop Road, Sheffield,S10 2JF, UK (e-mail: Gary.Mills@sth.nhs.uk). collapse and tidal recruitment, and with a small tidal volume to avoid end-inspiratory over distension. However, due to absence of a bedside monitoring technique, the choice of optimal ventilator titration strategy remains controversial.

Arterial blood gas analysis and the recording of PressureVolume (P-V) curve give only an indication of the overall lung function. Currently, chest Computed Tomography (CT scanner) is the most reliable technique for the clinical assessment of regional lung recruitment and ventilation distribution in patient with ARDS. By measuring lung densities, well-aerated regions can be easily differentiated from poorly and non-aerated lung regions. However, CT exposes the patient to radiations and is not a routine technique that is available at the bedside.

Electrical Impedance Tomography (EIT) is a promising noninvasive monitoring technique that allows real-time imaging of regional ventilation of the lungs at the bedside. EIT, however, has a low spatial resolution as compared to CT and Magnetic Resonance Imaging (MRI) and is sensitive to electrode placement.

The basic principle of EIT is based on the injection of small currents of about $5 \mathrm{~mA}$ between adjacent surface electrodes placed circumferentially around the thorax and measurement of the resulting potential differences. Since air is a poor conductor of electric current, lung tissue filled with air will have a low conductivity in contrast to water and blood. Images can be reconstructed and displayed in realtime to monitor conductivity changes resulting from the respiratory activity.

The first clinical EIT images were obtained from the Sheffield Group [5] who used a filtered version of the backprojection method used in X-ray CT imaging. Recently a software package (EIDORS) implementing different methods for the solution of the forward and inverse problems in EIT using finite elements modeling techniques has been made available for public use [6].

Many current ongoing research studies are being directed at demonstrating the ability of EIT to image regional lung ventilation in a clinical setting [7]-[10]. Comprehensive literature reviews in this field can be found in [11], [12].

The purpose of this paper is to demonstrate the principles of EIT imaging on a physiological model of the lung mechanics. The lung conductivity/volume relationship was modeled using published data recorded from EIT and spirometry measurements performed on a human subject [13]. A 2D finite element cross-sectional model of the thorax with sixteen electrodes was employed to simulate EIT measurements and reconstruct the conductivity distribution. 


\section{Simulation Model SetuP}

The simulation setup shown in Fig. 1 includes a model of the respiratory mechanics, a parametric model describing the relationship between the assumed lung volume $(V)$ and the left $\left(\sigma_{L}\right)$ and right $\left(\sigma_{R}\right)$ lung conductivities and a finite element cross section model of the thorax on which the EIT current injection and voltage measurements are performed. These simulated EIT data (assumed to be the 'real measurements') are then matched with the model predicted data set until some precision is reached.

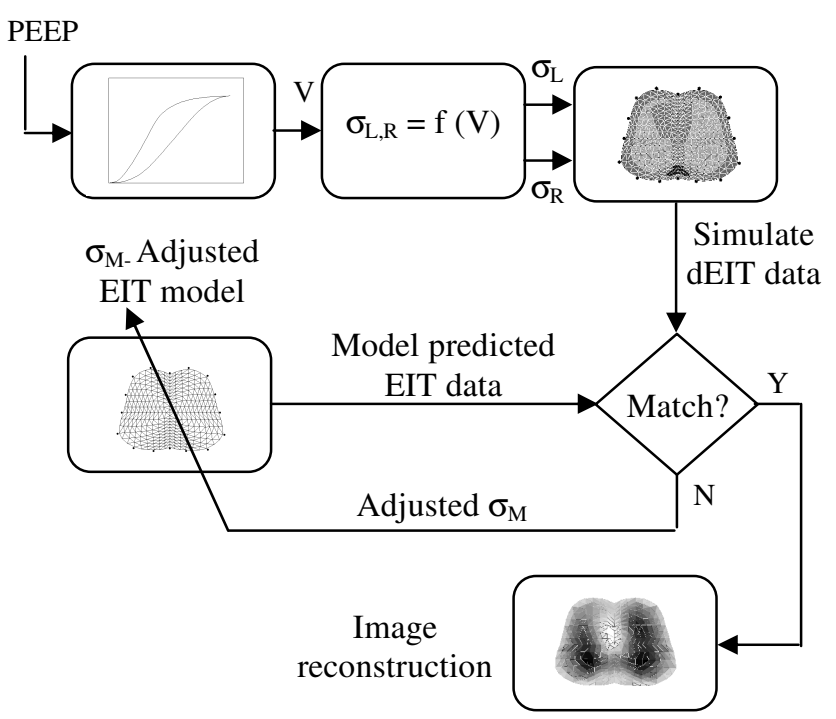

Fig.1. Structure and components of the simulation model. PEEP = Peak End-Expiratory Pressure, $V=$ lung volume, $\sigma_{L}$, $\sigma_{R}=$ left and right lung relative conductivities, $\sigma_{M}=$ EIT model conductivity.

A detailed description of these model components is presented in the following sections.

\section{A. Model of the Lung Mechanics}

A model based on simple physiology and lung mechanics has been proposed by Hickling [14], [15]. The model is based on the fact that recruitment and derecruitment of the lung alveoli are the principal cause of inflation and deflation respectively.

The lung is modeled as multiple units or alveoli which are distributed into compartments characterized by different superimposed pressure (gravitational pressure due to lung weight). In the upright position, the gravitational pressure increases linearly from the uppermost (independent region) compartment to the lowermost (dependant region) compartment. In the supine position the superimposed pressure increases from the ventral compartment (independent region) to the dorsal compartment (dependant region).

The lung units are described by their compliance curve which gives a nonlinear relationship between the applied pressure and the lung unit volume. The following equation is used to model this relationship [16]:

$$
V=V_{0}(1-\exp (-P \log (2 / h))
$$

Where $V$ is the lung volume, $V_{0}$ is the maximum volume at high pressure, $P$ is the pressure and $\mathrm{h}$ is the half-inflation pressure.

In the model, the lung unit can assume only two possible states: recruited (or open) and de-recruited (or closed) [14], [17]. These two states are governed only by the Threshold Opening Pressure (TOP) which the critical pressure above which the lung unit pops open and Threshold Closing Pressure (TCP) below which the unit collapses respectively. The model uses normally distributed TOP and TCP pressures with a specific mean and standard deviation [18] that may be adjusted to reflect the heterogeneous characteristic of alveoli under different abnormal lung conditions such as ARDS [19], [20]. Fig. 2 illustrates the mechanics of a single lung unit during inflation and Duftititioninflation (or expiration) when the applied pressure exceeds the TOP, the lung unit pops open and assumes a volume according to the PV equation (1). During deflation (expiration) when the applied pressure falls below the TCP, the lung unit collapses and its volume becomes zero.

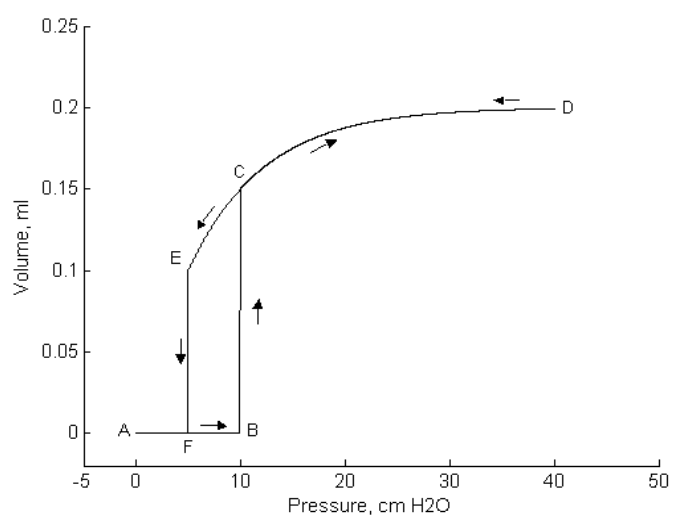

Fig.2. Mechanics of inflation (path ABCD) and deflation (path DEFA) of a single lung unit. TOP and TCP coincide with points $B$ and $F$ respectively.

The lung volume at a given pressure can then be calculated by adding up the contributions of the recruited lung units in the different compartments at that specific phressmredel parameters used throughout are listed in Table 1 [14].

TABLE I

LUNG MECHANICS MODEL BASELINE PARAMETERS

\begin{tabular}{lc}
\hline \hline \multicolumn{1}{c}{ Parameters } & Value \\
& \\
\hline Number of alveoli per compartment & 9000 \\
Number of compartments & 30 \\
Gravitational pressure & 0 to $14.5 \mathrm{cmH}_{2} \mathrm{O}$ \\
Lung volume (litres) & 6 \\
$\mathrm{~V}_{0}$ (litres) & 3.8 \\
$\mathrm{~h}$ & 5 \\
\hline \hline
\end{tabular}




\section{B. Model of the Conductivity-Lung Volume Relationship}

This parametric model provides a link between the lung mechanics and EIT models. It defines the relationship between changes in the basal conductivity of the lungs and the inspired fraction of air. The data used to derive this model were obtained from EIT and spirometry measurements recorded from a human subject during a respiratory cycle [13]. The left $\left(\sigma_{L}\right)$ and right $\left(\sigma_{R}\right)$ lung relative conductivities estimated from a thorax model for different inspiration levels are listed in Table 2 [13]. The inspiration fraction $F$ is defined as: $F=\left(V_{\text {insp }}-V_{\min }\right) /\left(V_{\max }-\right.$ $\left.V_{\min }\right)$. Where $V_{\text {insp }}$ represents the tidal volume, $V_{\min }$ and $V_{\max }$ are respectively the minimum and maximum volumes assumed during tidal breathing.

TABLE II

LEFT AND RIGHT LUNG CONDUCTIVITIES $\left(\sigma_{L}, \sigma_{R}\right)$ FOR DIFFERENT INSPIRATION FRACTION $(F)$

\begin{tabular}{ccc}
\hline$F(\%)$ & $\sigma_{L}$ & $\sigma_{R}$ \\
\hline 0 & 0.8 & 0.8 \\
20 & 1.0 & 0.8 \\
40 & 0.9 & 0.7 \\
60 & 0.7 & 0.6 \\
80 & 0.5 & 0.6 \\
100 & 0.4 & 0.5 \\
\hline \hline
\end{tabular}

Fitting these discrete values to a third order polynomial gives the parametric model plotted in Fig. 3.
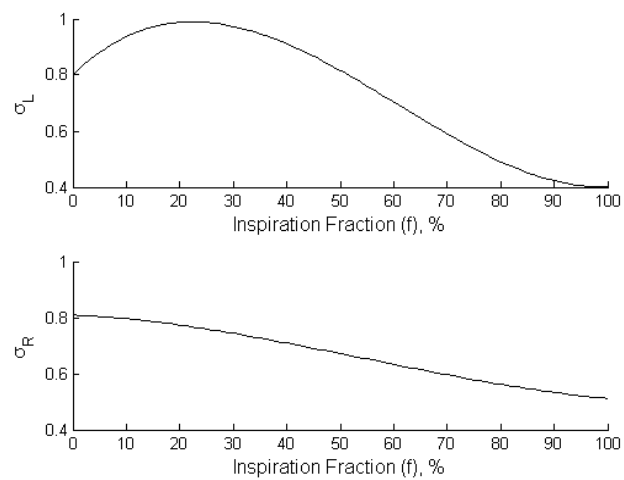

Fig. 3. Lung conductivities $\sigma_{L}$ and $\sigma_{R}$ as a function of $F$.

\section{Model of EIT}

In EIT, current patterns are injected into the body via surface electrodes and boundary voltages are measured to reconstruct a cross-sectional image of internal distribution of the conductivity or resistivity. The quality of reconstructed images depends on (i) the number of electrodes and data collection strategy and (ii) the reconstruction algorithm employed. The most popular data collection strategy is the so-called adjacent or four-electrode whereas the type of reconstruction algorithm ranges from the simplest linearised single-step method to a computationally intensive iterative techniques.

Mathematically, the problem of recovering the conductivity or resistivity within the body from the surface measurements of currents and potentials is a nonlinear inverse problem and severely ill-posed. At low frequencies, these quantities are related by Laplace's equation and Newman type boundary condition:

$$
\begin{aligned}
& \nabla \cdot(\sigma \nabla u)=0 \\
& \sigma \frac{\partial u}{\partial \vec{n}}=\left\{\begin{array}{lr}
\vec{J} & \text { under the electrodes } \\
0 & \text { elsewhere }
\end{array}\right.
\end{aligned}
$$

Where $\sigma$ is the conductivity, $u$ is is the potential, $\vec{J}$ is the density of the injected current and $\vec{n}$ is the normal vector to the surface. For the uniqueness of the solution of (2), a variety of assumptions have to be made [18]. To solve the inverse problem one needs to solve the forward problem for some assumed conductivity distribution and compare the generated voltages with those obtained from real measurements. The finite element method was employed for the numerical solution of (2) by subdividing the 2D crosssection of the thorax into a finite number of triangular elements. The electric potential is expressed as a linear combination of the nodal basis functions. Each element is assumed to be homogenous and having the same conductivity. The Finite Element Model (FEM) shown in Fig. 4 was used to simulate the subject's cross section of the thorax. The FEM was divided into four regions of different conductivities which were fixed to their basal values except those of the left and right lung that were varied according to Fig. 3. EIT numerical solution involves updating the conductivity distribution until the predicted and measured voltages match each other within a desired accuracy. The Gauss-Newton iterative algorithm implemented in [6] was used in this work.

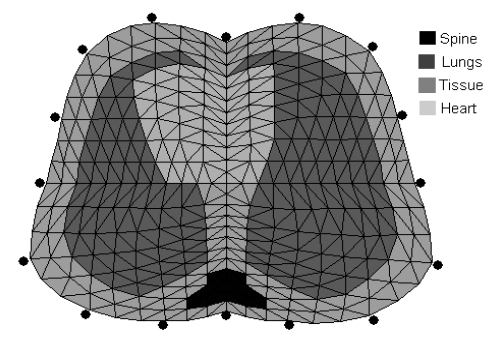

Fig. 4. Thorax FEM model with 576 elements, 313 nodes and 16 electrodes.

\section{SIMULATION RESUlTS}

In ARDS, the lungs become stiffer and the lung units tend to open and collapse at higher pressures compared with normal lung. In the model, the Mean and Standard Deviation (SD) of TOP and TCP pressures were given the values shown in Table 2 to simulate different intensities of ARDS [19], [21]. The Mean indicates the pressure at which the maximum of recruitment (TOP) and derecruitment (TCP) of lung units occurs, whereas SD describes the spread of the lung units' population with respect to the TOP and TCP. 
Moreover the Functional Residual Capacity (FRC), that is amount of air that stays in the lungs during normal is also reduced in ARDS affected lungs. Estimated values FRC under the degrees of ARDS conditions considered are given in Table 2.

TABLE II

TOP AND TCP PRESSURES

FOR THE SIMULATED ARDS SCENARIOS

\begin{tabular}{cccccc}
\hline \hline Degree of & \multicolumn{2}{c}{ TOP $\left(\mathrm{cmH}_{2} \mathrm{O}\right)$} & \multicolumn{2}{c}{ TCP $\left(\mathrm{cmH}_{2} \mathrm{O}\right)$} & FRC (litres) \\
ARDS & Mean & SD & Mean & SD & \\
\hline normal & 4.5 & 2 & 2 & 2 & 2.4 \\
mild & 10 & 2.9 & 2.5 & 2.4 & 2.2 \\
moderate & 14.5 & 3.8 & 4.5 & 2.9 & 1.8 \\
severe & 24.5 & 4.8 & 13 & 3.8 & 1.5 \\
\hline \hline
\end{tabular}

Fig. 5 shows the plots of the Gaussian distributions of TOP and TCP pressures for the classes of simulated lung conditions given in Table 2.

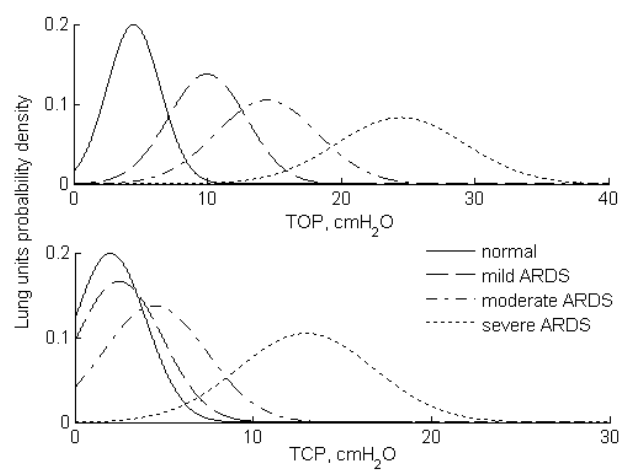

Fig. 5. Gaussian distribution of TOP and TCP of normal and ARDS conditions (mild, moderate and severe).

A tidal breathing cycle is simulated by traversing up (inflation) and then down (deflation) the airway pressure range in small steps from PEEP to PIP (Peak Inspiratory Pressure) and then from PIP to PEEP respectively.

Fig. 6 shows the PV curves obtained for healthy and ARDS affected lungs. PEEP and PIP were set to 0 and $40 \mathrm{cmH}_{2} 0$ respectively.
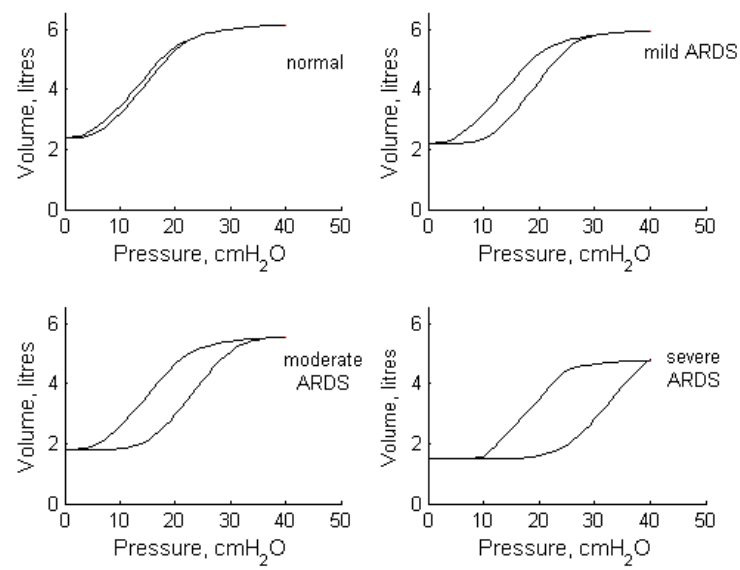

Fig. 6. Pressure-volume curves for the simulated normal (healthy) and ARDS conditions (mild, moderate and severe).
In ARDS, the PV curves are right-shifted and characterized by larger hysteresis between the inflation and deflation limbs which is reflected by the difference in TOP and TCP pressures.

Fig. 7 presents the simulations of the severe ARDS model for four different values of PEEP $(10,12,14$, and 16 $\left.\mathrm{cmH}_{2} \mathrm{O}\right)$ under constant PIP $\left(40 \mathrm{cmH}_{2} \mathrm{O}\right)$. Higher PEEP values lead to higher derecruitment volume which accounts for the lung units that are aerated at end-inspiration and remain open during expiration.
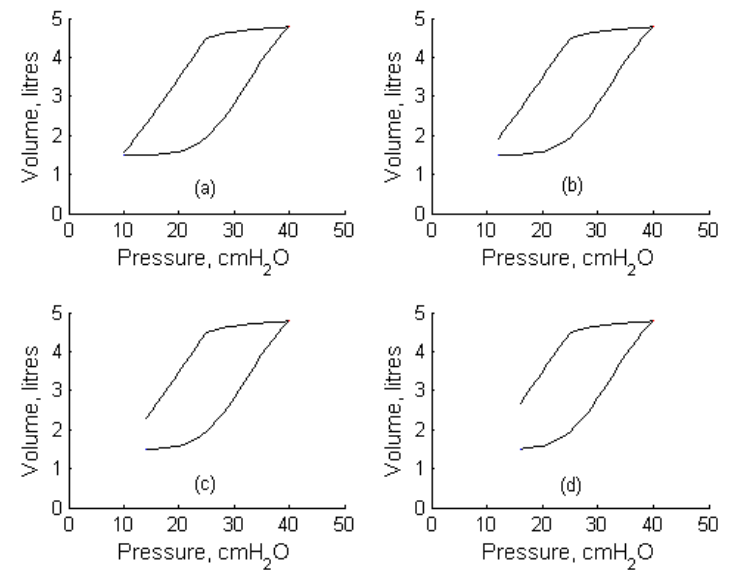

Fig. 7. PV curves of the simulated severe ARDS lung with $\mathrm{PEEP}=10 \mathrm{cmH}_{2} \mathrm{O}$ (a), $12 \mathrm{cmH}_{2} \mathrm{O}$ (b) $14 \mathrm{cmH}_{2} \mathrm{O}$ (c) and 16 $\mathrm{cmH}_{2} \mathrm{O}(\mathrm{d})$.

The physiological model of the lung mechanics simulating different degrees of ARDS conditions is incorporated with the EIT model in the simulation setup of FigAdain, the model is cycled through the assigned airway pressure and at each pressure step the calculated lung volume is used to set the new conductivities of the left and right lungs on the thorax model of Fig. 4. EIT data are then generated using adjacent drive patterns with an injected current of $5 \mathrm{~mA}$. These measured EIT data are compared to those predicted from another FEM model using the same drive and measurement patterns until some desired accuracy has been achieved. On convergence, the reconstructed image of the lung conductivity distribution is displayed. The image sequence is obtained by calculating conductivity changes from a reference state which defines the basal conductivities for the lungs and the rest the organs to a new state. This is the so-called difference imaging the EIT terminology as compared to absolute image which tries to recover the absolute value of the conductivity or resistivity from the boundary measurements.

Figs. 8 and 9 show the sequence of lung image slices (progressing from left to right and top to bottom) reconstructed from a simulated breath related to the and severe ARDS models with the ventilator PEEP and PIP set to 0 and $40 \mathrm{cmH}_{2} \mathrm{O}$ respectively. Clear lung regions correspond to expiration at FRC and dark regions to inspiration. This image contrast is the result of the 
simulated changes in the conductivity of the left and right lungs. Lung inflation begins at slice 6 .

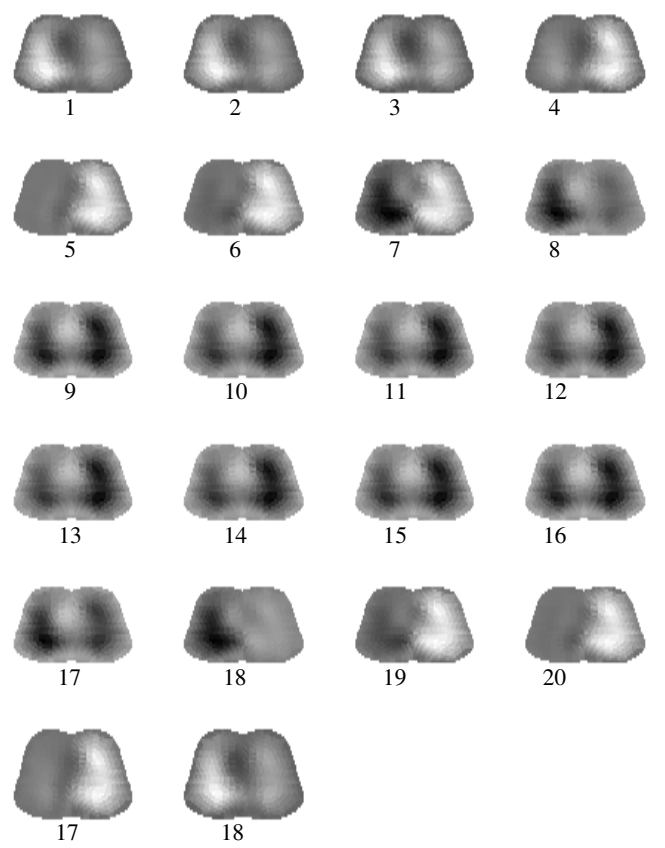

Fig. 8. Temporal lung image slices of a breath (expirationinspiration-expiration) related to moderate ARDS model. $\mathrm{PEEP}=0 \mathrm{cmH}_{2} \mathrm{O}, \mathrm{PIP}=40 \mathrm{cmH}_{2} \mathrm{O}$.

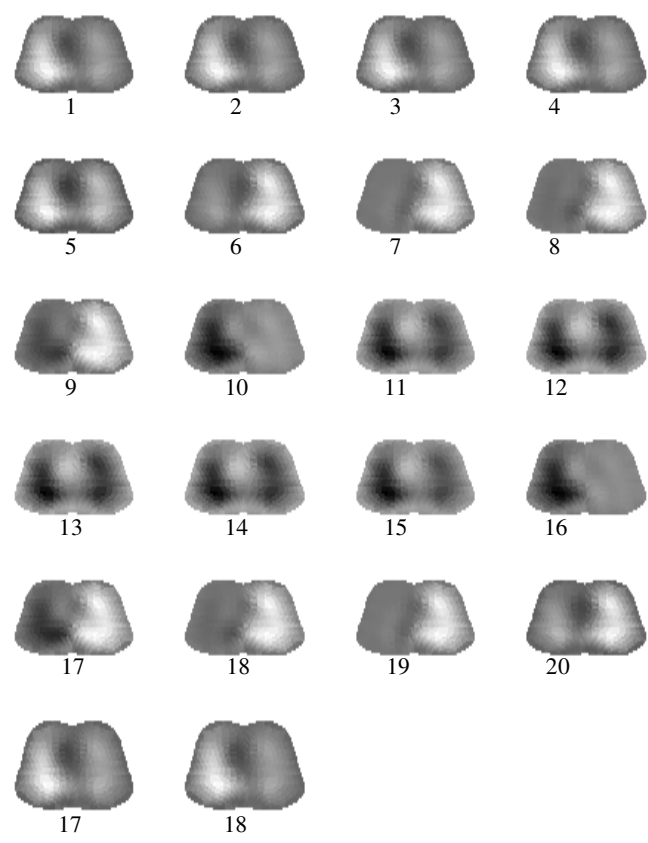

Fig. 9. Temporal lung image slices of a breath (expirationinspiration-expiration) related to severe ARDS model. PEEP $=0 \mathrm{cmH}_{2} \mathrm{O}, \mathrm{PIP}=40 \mathrm{cmH}_{2} \mathrm{O}$.

In the moderate ARDS model, lung inflation has begun at slice number 6 , whereas in the case of severe ARDS model has commenced at slice 8 confirming the results obtained with the static PV curves of Fig. 6 and the fact that TOP pressures of the lung units in the case of severe ARDS are higher than in moderate ARDS model

Figs. 10 and 11 show the effect of PEEP on these models of ARDS lungs. PEEP was set to $10 \mathrm{cmH}_{2} \mathrm{O}$ with all the other parameters maintained to their previous values. Lung inflation starts at slice 6 and recruitment is maintained for those lung units whose TCP pressures are below the PEEP value selected. This PEEP value resulted in a larger derecruitment volume, which is the lung expansion at the end of expiration, in the moderate ARDS model than in the severe again due to the difference in the distribution of TCP pressures in these two models (Table 2).

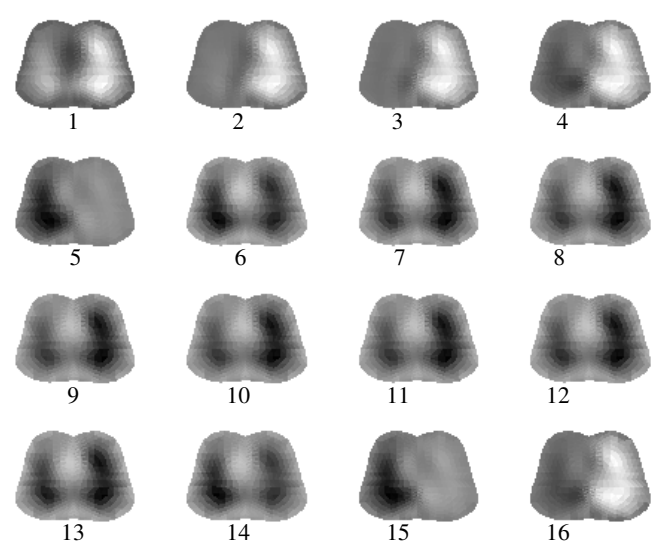

Fig. 10. Image slices of a respiratory cycle related to moderate ARDS model. PEEP $=10 \mathrm{cmH}_{2} \mathrm{O}, \mathrm{PIP}=40$ $\mathrm{cmH}_{2} \mathrm{O}$.

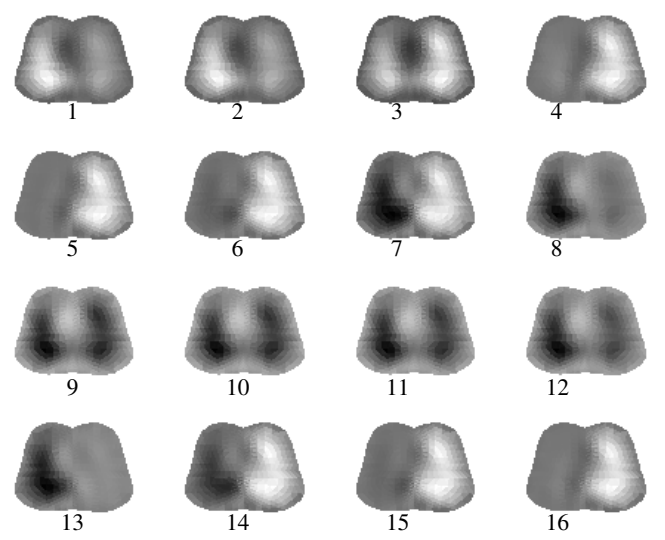

Fig. 11. Temporal lung image slices of a breath (expirationinspiration-expiration) related to severe ARDS model. PEEP $=0 \mathrm{cmH}_{2} \mathrm{O}, \mathrm{PIP}=40 \mathrm{cmH}_{2} \mathrm{O}$.

Interestingly, these simulation results demonstrate that EIT is able to continuously track ventilation distribution in the lungs, and thus can be effectively used to adjust the level of PEEP that is sufficient to prevent alveolar collapse during lung deflation. 


\section{CONCLUSION}

EIT is an established monitoring technique with the potential of becoming a valuable bedside tool for the assessment of the pulmonary function. EIT is capable of tracking local changes in pulmonary air contents and thus, can be used to continuously guide a proper titration of PEEP in the care of ARDS patients.

A simulation model based on respiratory physiology has been developed to demonstrate the principles of EIT monitoring on ARDS affected lung models under mechanical ventilation. The model is not intended to be a perfect and complete representation of the respiratory physiology of ventilated patients affected by ARDS. However, while relatively simple, it gives a good understanding of the processes of recruitment and derecruitment in ARDS affected lungs and illustrates how ventilator settings can be optimized with the aid of EIT

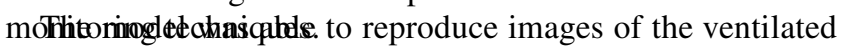
lung under different ARDS conditions which were consistent and presented a good agreement with the pressure-volume curve simulation results obtained for the same model of ARDS lungs. A rather simplistic parametric model has been used here to relate left and right lung conductivities to lung volume; however, more comprehensive models that would take into account the effect of anthropometric variables, gender and posture can be used.

\section{ACKNOWLEDGEMENT}

The authors gratefully acknowledge the financial support of the UK Engineering and Physical Sciences Research Council (EPSRC) under Grant EP/520807/1.

\section{REFERENCES}

[1] G. R. Bernard, "Acute respiratory distress syndrome: A historical perspective," Am J Resp Crit Care Med, vol 172, pp 798-806, 2005

[2] A. B. Weinacker and L. T. Vaszar, "Acute respiratory distress syndrome: Physiology and new management strategy," Ann Rev Med, 52, pp. 221-237, 2001.

[3] S. Grasso, V. Fanelli, A. Cafarelli, R. Anaclerio, M. Amabile, G. Ancona, and T. Fiore, "Effects of high versus low positive endexpiratory pressures in acute respiratory distress syndrome," Am. J. Respir. Crit. Care Med., 171(9), pp.1002 - 1008, May 2005.

[4] A. Malhorta, "Tidal volume ventilation in the acute respiratory distress syndrome," N Engl J Med, 357(11), pp. 1113-1120, Sept 2007.

[5] B. H. Brown, D. C. Barber, and A.D. Seager, "Applied potential tomography: possible clinical," Clin Phys Physiol Mea., 6, pp. 109$121,1985$.

[6] A. Adler and W. R. B. Lionheart, "Uses and abuses of EIDORS: an extensible softare base for EIT," Physiol Meas., 27, pp. S25-S42, 2006. Available: http://eidors3d.sourceforge.net/).

[7] I. Frerichs, G. Schmitz, S. Pulletz, D. Schädler, G. Zick, J. Scholz, and N. Weiler, "Reproducibility of regional lung ventilation distribution determined by electrical impedance tomography during mechanical ventilation," Physio Meas, 28, pp. S261-S267, 2007.

[8] C. Putensen, H. Wrigge, and N. Weiler, "Electrical impedance tomography guided ventilation therapy," Current Opinion in Critical Care, 13(3), pp. 344-350, 2007.
[9] J. Hinz, O. Moerer, P. Newmann, T. Dudykevych, G. Hellige, and M. Quintel, "Effect of positive end-expiratory-pressure on regional ventilation in patients with acute lung injury evaluated by electrical impedance tomography," European J of Anaesthesiology, 22, pp. 817$825,2005$.

[10] J. A. Victorino, J. B. Borges, V. N. Okamoto, G. F. J. Matos, M. R. Tucci, M. P. R. Caramez, H. Tanaka, F. S. Sipmann, D. C. B. Santos, C. S. V. Barbas, C. R. R. Carvalho, and M. B. P. Amato, "Imbalances in regional lung ventilation: A validation study on electrical impedance tomography," Am J Respir Crit Care Med, 169, pp. 791$800,2004$.

[11] I. Frerichs, "Electrical impedance tomography (EIT) in applications related to lung and ventilation: A review of experimental and clinical activities,"Physiol Meas, 21,, pp. R1-R21, 2000.

[12] G. Panoutsos, M. Mahfouf, B. H. Brown, and G. H. Mills, "Electrical impedance tomography (EIT) in pulmonary measurement: a review of applications and research," Proceedings of the $5^{\text {th }}$ IASTED International Conference: biomedical engineering, Innsbruck, Austria, pp. 221-230, 2007.

[13] L. A. Smulders, and A. van Oosterom, "Application of electrical impedance tomography to the determination of lung volume," Clin Phys Physiol Meas, 13, pp. 167-170, 1992.

[14] K. G. Hickling, "The pressure-volume curve is greatly modified by recruitment: A mathematical model of ARSD lungs," Am J Respir Crit Care Med, 158, pp. 194-202, 1998.

[15] K. G. Hickling, "Best compliance during a decremental, but not incremented, positive end-expiratory pressure trial is related to openlung positive en-expiratory pressure: A mathematical modelof acute respiratory distress syndrome lungs," Am J Respir Crit Car Med,163, pp. 69-77, 2001.

[16] E. Salazar, and J. H. Knowles, "An analysis of the pressure-volume characteristics of the lungs," Journal of Applied Physiol, 19, pp. 97104, 1964.

[17] T. Yuta, J. G. Chase, G. M. Shaw, and C. Hann, "Dynamic models of ARDS lung mechanics for optimal patient ventilation," Proceedings of the $26^{\text {th }}$ Annual International Conference: of the IEEE EMBS San Francisco, USA, pp. 861-864, Sept 2004.

[18] D. S. Holder, "Electrical Impedance Tomography: Methods, History and Applications," Series in Medical Physics and Biomedical Engineering, Taylor \& Francis, 2004.

[19] S. Crotti, D. Mascheroni, P. Caironi, P. Pelosi, G. Ronsoni, M. Mondino, J. J. Marini, and L. Gattinoni, "Recruitment and Derecruitment during Acute Respiratory Distress Syndrome," Am J Respir Crit Care Med, , 164, pp. 131-140, 2001.

[20] D.G. Markhorst, H. R. van Genderingen, and A.J. van Vught, "Static pressure-volume curve characteristics are moderate estimators of optimal airway pressures in a mathematical model of (primary/pulmonary) acute respiratory distress syndrome," Intensive Care Med, 30, pp. 2086-2093, 2004.

[21] T. Yuta, "Minimal model of lung mechanics for optimising Ventilator therapy in critical care," PhD Dissertation, University of Canterbury,Christchurch, New Zealand, 2007. 